\title{
Toward photonic crystal based spatial filters with wide angle ranges of total transmission
}

A. E. Serebryannikov, A. Y. Petrov, and Ekmel Ozbay

Citation: Appl. Phys. Lett. 94, 181101 (2009);

View online: https://doi.org/10.1063/1.3127443

View Table of Contents: http://aip.scitation.org/toc/apl/94/18

Published by the American Institute of Physics

\section{Articles you may be interested in}

Spatial filtering using dielectric photonic crystals at beam-type excitation

Journal of Applied Physics 108, 113106 (2010); 10.1063/1.3498810

Super-collimation by axisymmetric photonic crystals

Applied Physics Letters 104, 221108 (2014); 10.1063/1.4881839

Spatial filtering with photonic crystals

Applied Physics Reviews 2, 011102 (2015); 10.1063/1.4907345

Omnidirectional gap and defect mode of one-dimensional photonic crystals containing negative-index materials

Applied Physics Letters 83, 5386 (2003); 10.1063/1.1637452

Self-collimating phenomena in photonic crystals

Applied Physics Letters 74, 1212 (1999); 10.1063/1.123502

Intrinsic beam shaping mechanism in spatially modulated broad area semiconductor amplifiers

Applied Physics Letters 103, 132101 (2013); 10.1063/1.4821251

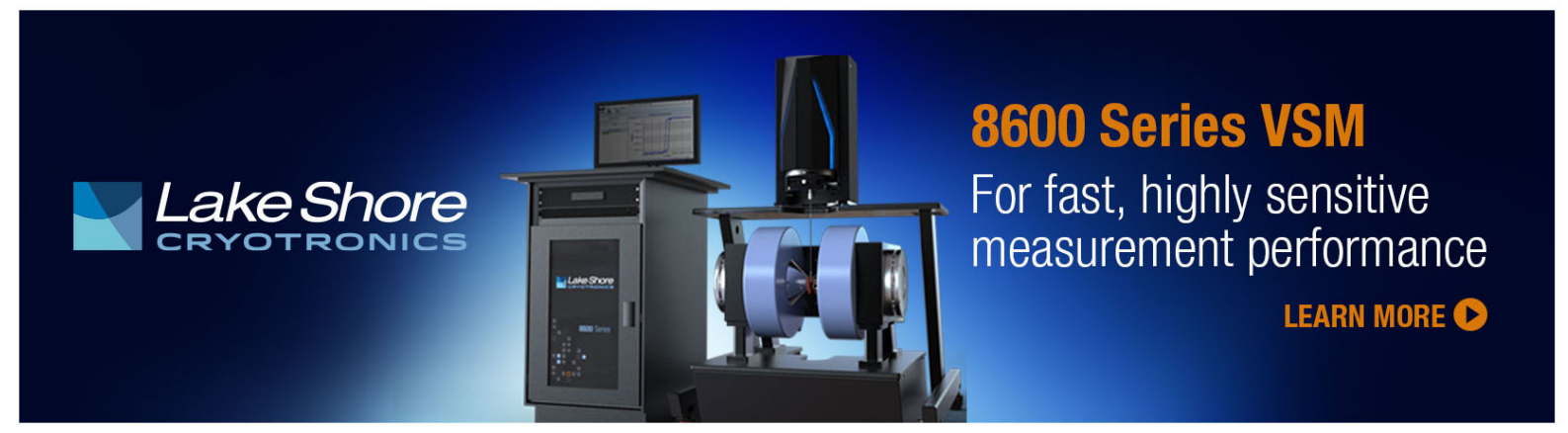




\title{
Toward photonic crystal based spatial filters with wide angle ranges of total transmission
}

\author{
A. E. Serebryannikov, ${ }^{1,2, a)}$ A. Y. Petrov, ${ }^{2}$ and Ekmel Ozbay ${ }^{1}$ \\ ${ }^{1}$ Department of Physics, Department of Electrical and Electronics Engineering, Nanotechnology Research \\ Center-NANOTAM, Bilkent University, 06800 Ankara, Turkey \\ ${ }^{2}$ Technische Universitaet Hamburg-Harburg, D-21071 Hamburg, Germany
}

(Received 31 August 2008; accepted 8 April 2009; published online 5 May 2009)

\begin{abstract}
Spatial filters with steep switching between wide ranges of total transmission and total reflection can be obtained by using two-dimensional dielectric photonic crystals, which are a few wavelengths thick. The guidelines for engineering bandpass and bandstop filters are given. The flatness of isofrequency contours that are localized around a periphery point of the first Brillouin zone is a necessary but insufficient condition for the existence of wide angle ranges of total transmission at intermediate and large angles of incidence. Such ranges that are wider than $20^{\circ}$ are demonstrated. () 2009 American Institute of Physics. [DOI: 10.1063/1.3127443]
\end{abstract}

Spatial filters have many applications that are related to information processing and image enhancement. They are used for the analysis and modification of a spatial spectrum, radar data processing, aerial imaging, the detection of extrasolar planets, as well as in biomedical applications, e.g., see Refs. 1 and 2 and the references therein. Simultaneous spatial and frequency domain filtering is required for controlling laser radiation. ${ }^{3}$ The known implementations of spatial filters include anisotropic media, ${ }^{1}$ resonant grating systems, ${ }^{3,4}$ interference patterns, ${ }^{5}$ multilayer stacks combined with a prism, ${ }^{2}$ metallic grids over a ground plane, ${ }^{6}$ and slabs of photonic crystal (PC) with defects. ${ }^{7}$ The possibilities for the realization of narrow bandpass filters ${ }^{3,4,7}$ and low-pass, high-pass, and wide bandpass filters with a rather steep switching between pass- and stopbands ${ }^{1,2}$ have been demonstrated. In Ref. 1, nonideal high-pass filters with the adjacent ranges of transmittance $T \leq 1$ and reflectance $R=1$ have been obtained using the anticutoff media, which are realized due to different signs of the elements of the permittivity and permeability tensors. However, the problem still exists concerning the possibility of obtaining wide angle domain ranges of total transmission. Besides, dispersion features of the anticutoff media do not allow realizing truly bandpass filters and bandstop filters at fixed sign of the angle of incidence $\theta$, while those typical for some PCs can be appropriate for this purpose.

In the present paper, we will show that wide adjacent ranges of variation of $\theta>0$ with $T \approx 1$ and $R=1$, and with a steep switching between them can be realized using twodimensional, square-lattice, dielectric PCs with proper topologies of isofrequency contours (IFCs). The theoretical study is carried out in the case when an electric field is parallel to a rod axis (TM polarization). The presented transmission results are obtained numerically by using a rigorous integral equation technique. To calculate dispersion, CST MICROWAVE STUDIO software and a self-made post-processing code have been used.

Figure 1 shows the examples of IFCs in $\mathbf{k}$ space, which are associated with high-pass, bandpass, and bandstop filters.

\footnotetext{
${ }^{\text {a) }}$ Author to whom correspondence should be addressed. Electronic mail: serebryannikov@tu-harburg.de.
}

To obtain a high-pass or bandpass filter at $\operatorname{sgn} \theta=$ const, IFCs have to be localized around either the $\mathrm{M}$ point of the first Brillouin zone (FBZ) if the PC interfaces are parallel to the $\Gamma$-X direction [case (a)], or the $\mathrm{X}$ point if they are parallel to the $\Gamma$-M direction [case (b)]. To realize a bandstop or dualbandpass filter at $\operatorname{sgn} \theta=$ const, that has a passband including $\theta=0$, IFCs should be localized around the M point while the interfaces are parallel to the $\Gamma-\mathrm{M}$ direction [case (c)], or the IFCs localized around the $\Gamma$ point should coexist with those around the $\mathrm{M}$ point while the interfaces are parallel to the $\Gamma$-X direction [case (d)].

In Figs. 1(a), 1(c), and 1(d), transmission at large and/or intermediate $\theta$ can occur if $k_{0}>k_{\text {min }}^{\mathrm{M}}$. Here, $k_{0}=\left|\mathbf{k}_{0}\right|, \mathbf{k}_{0}$ is the wave vector of the incident wave, $k_{\min }^{\mathrm{M}}=\min k_{x}^{\mathrm{M}}$, and $k_{x}^{\mathrm{M}}$ is the wave vector component of the Floquet-Bloch (FB) wave, which is parallel to the PC interfaces and corresponds to IFCs around the M point. It is seen that a bandpass filter can be obtained in case (a) at $k_{0} a>2 \pi-k_{\min }^{\mathrm{M}} a$, where $a$ is lattice constant, or even at a less strict condition. Throughout the paper, $\varepsilon_{r}, \varepsilon_{h}=1$, and $d$ mean permittivities of the rods and host medium, and diameter of the rods, respectively.

A required wide $\theta$-domain passband, which appears at large and/or intermediate $\theta$, must satisfy the following conditions at a fixed frequency:
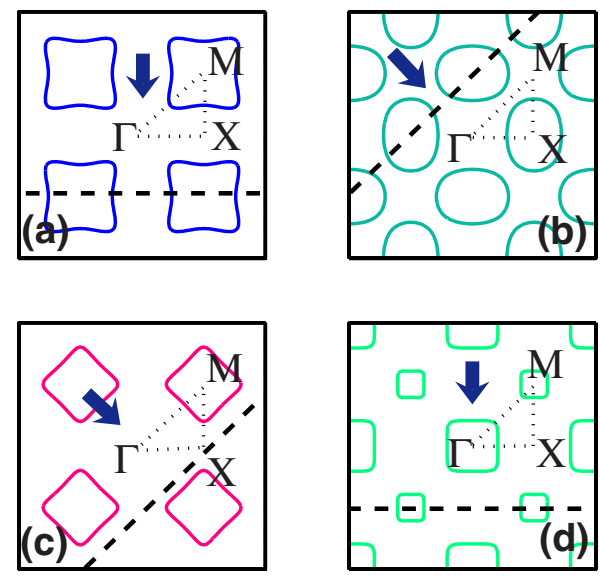

FIG. 1. (Color online) Examples of IFCs for a square-lattice PC: arrowsdirection of the incident wave at $\theta=0$, dashed lines-orientation of the interfaces, dotted lines-FBZ boundaries. 


$$
T(\theta)=1, \quad \theta_{b} / \theta_{s} \gg 1,
$$

where $\theta_{1}<\theta<\theta_{2}, \theta_{1}>0, \theta_{2} \leq \pi / 2, \theta_{b}=\theta_{2^{-}} \theta_{1}, \theta_{b}$ is the passband width, and $\theta_{s}$ is the width of the switching range. It is noteworthy that the conditions (1) are inconsistent with the typical features of the conventional Fabry-Pérot resonances [e.g., $T(\theta) \neq$ const], in terms of which the total transmission through PCs is often interpreted. ${ }^{7,8}$

In the context of our present interest, it is worth mentioning the omnidirectional filters based on the use of defect modes in one-dimensional PCs, which are composed of the alternating layers of $\mu$-negative and $\varepsilon$-negative metamaterials, ${ }^{9}$ or double-negative and double-positive metamaterials. ${ }^{10}$ An exotic Fabry-Pérot type behavior has been realized there so that the same resonance remains for all $\theta$. According to Ref. 11, $\theta$ insensitive Fabry-Pérot type resonances can appear in PCs, provided that the IFCs are flat. In contrast to the canalization ${ }^{11}$ and superlens ${ }^{12,13}$ regimes, where IFCs for PC must be wider than for air and flat for any $\theta$, we require here that IFCs are flat at $\theta_{1}<\theta<\theta_{2}$. Hence, the necessary conditions of the wide ranges of $T=1$ present a combination of the requirement of IFCs flatness and a requirement of the IFCs topology, according to Fig. 1. At the same time, the sign of the product $S \cdot \mathbf{k}$ ( $\boldsymbol{S}$ is the Poynting vector and $\mathbf{k}$ is the wave vector of a FB wave), which determines the handedness, is expected to exert no effect on the possible existence of the ranges with $d T / d \theta \approx 0$.

The above-mentioned requirements have to be tolerant with respect to a $k_{0} a$ variation. In the conventional FabryPérot resonators, the resonances appear at $n^{(f)} k_{0} D \cos \theta$ $=\pi m, m=1,2, \ldots$, where $D$ and $n^{(f)}$ are the distance between the mirrors and the index of refraction of the filling medium, respectively. Hence, $T=1$ cannot be achieved at any fixed $k_{0} D$ and $\theta$. On the other hand, the resonance condition in PCs can be written as $|\mathbf{k}| D_{\mathrm{PC}} \cos \theta=\pi m$, where $D_{\mathrm{PC}}$ is $\mathrm{PC}$ thickness and $|\mathbf{k}| \cos \theta$ stays constant at varying $\theta$ due to IFC flatness. Because of a finite width of the $k_{0} a$ range where the IFCs are (near-)flat, the number of resonances with $T \approx 1$ within this range can depend on $D_{\mathrm{PC}}$. For example, a sole resonance can be achieved for a larger number of the rod
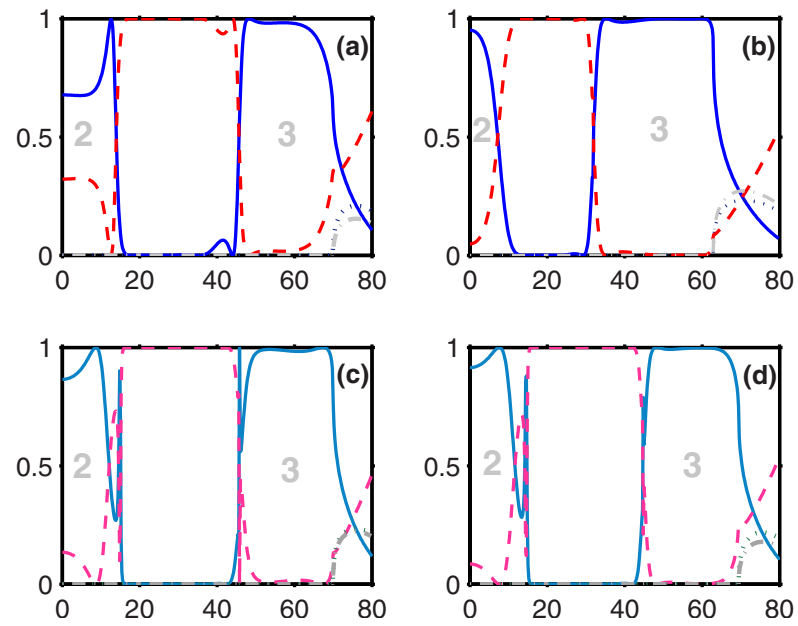

FIG. 2. (Color online) Zero-order transmittance $t_{0}$ (solid lines) and reflectance $r_{0}$ (dashed lines) and negative-first-order transmittance $t_{-1}$ (dotted lines) and reflectance $r_{-1}$ (dash-dotted lines) vs $\theta$ (in degrees) for PC with $d / a=0.4$ and $\varepsilon_{r}=11.4 ; k_{0} a=3.24$ (a,c), $k_{0} a=3.3325$ (b), $k_{0} a=3.245$ (d); $N=5[(\mathrm{a})$ and (b) $]$ and $N=8[(\mathrm{c})$ and (d) $] ; 2$ and 3-mode numbers of the FB waves.

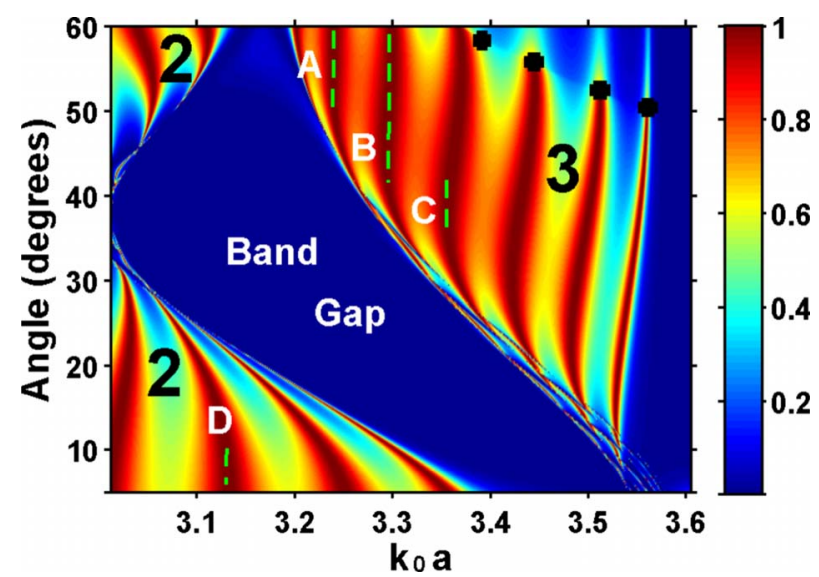

FIG. 3. (Color online) Zero-order transmittance at varying $k_{0} a$ and $\theta$ for $d / a=0.4, \varepsilon_{r}=11.4$, and $N=8 ; 2$ and 3 -mode numbers of the FB waves. The vertical dashed lines correspond to $T \approx 1$. The black crosses show the boundary of the negative-first-order transmission zone.

layers $N=N_{1}$ and $D_{\mathrm{PC}}=N_{1} a$, while no resonance appears within the same $k_{0} a$ range for smaller $N=N_{2}$ and $D_{\mathrm{PC}}=N_{2} a$. On the other hand, the use of a lower-order FB wave with (near-)flat IFCs should not necessarily lead to smaller $D_{\mathrm{PC}}$ at fixed $k_{0}$, since a larger $N$ can be required.

Several cases with near-flat IFCs have been considered. An example is shown in Fig. 2 for PCs with $\Gamma-X$ as an excitation interface and a similar IFC shape as in Fig. 1(d). One can see that a passband with $\theta_{b}>20^{\circ}$ can be obtained. The extent to which $T$ deviates from 1 within a passband and sharpness of passband boundaries depend on $N$ and $k_{0} a$. $D_{\mathrm{PC}}$ is typically a few wavelengths thick, e.g., $D_{\mathrm{PC}} / \lambda \approx 2.58$ in Fig. 2(a). The main problem arising when trying to realize a bandstop or dual-bandpass filter concerns the obtaining of two wide ranges of $T \approx 1$ at small and large/intermediate $\theta$ for the same $k_{0} a$. In Fig. $3, t_{0}$ is presented at a simultaneous variation of $k_{0} a$ and $\theta$. A, B, C, and D stand for the cases, in which conditions (1) are satisfied. In particular, case A corresponds to the larger- $\theta$ passband in Fig. 2(c). The "valleys" and "mountains" are nearly equidistant, indicating

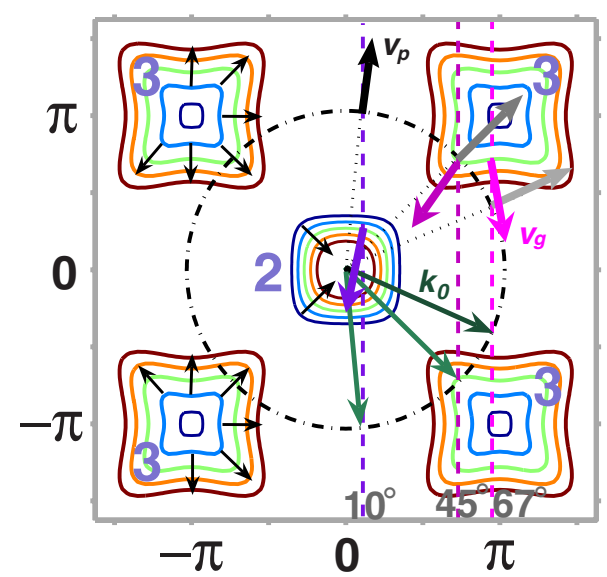

FIG. 4. (Color online) IFCs for PC with $d / a=0.4$ and $\varepsilon_{r}=11.4$ at $k_{0} a$ $=3.16,3.2,3.24,3.28$, and 3.32; IFC in air (dash-dotted circle) and the construction lines (dashed lines) are shown for $k_{0} a=3.24$ at $\theta=10^{\circ}, 45^{\circ}$, and $67^{\circ}$; directions of group velocity $\mathbf{v}_{g}$ and phase velocity $\mathbf{v}_{p}$ are shown by the most thick arrows directed toward the smaller and larger ordinate values, respectively; $\mathbf{k}_{0}$-midthick arrows; gradients of $\mathbf{v}_{g}$-thin arrows; 2 and 3 -mode numbers of FB waves. 
some similarity with the conventional Fabry-Pérot resonances.

The IFCs are shown in Fig. 4 for $k_{0} a$ values, which are either equal or rather close to those in cases A and B. The construction lines correspond to the vicinity of the edges of the passband in case $\mathrm{A}$, and to the small- $\theta$ passband $(0.86$ $<T<1$ ) at the same $k_{0} a$. Comparing Figs. 2-4, one can see that the wide intermediate- $\theta$ passband appears due to the third FB wave, for which the near-flat IFCs are located at the M point, showing $S \cdot \mathbf{k}<0$. In Fig. 4 , refraction is negative at $\theta=45^{\circ}$, i.e., at the left boundary of the passband, and positive within most part of the passband, including $\theta=67^{\circ}$ at the right boundary. The negative first diffraction order is propagating at $\theta>69.7^{\circ}$ so that the positively (zero-order) and negatively (negative-first-order) refracted beams may coexist. It is seen that the appearance of the upper boundary of the large/intermediate- $\theta$ passband is connected with the fact that the negative-first-order beam becomes propagating and with the reflection enhancement. Therefore, a passband, which is associated with a bandpass filter rather than with a high-pass one, can be obtained even at

$$
k_{\min }^{\mathrm{M}}<k_{0}<2 \pi / b-k_{\min }^{\mathrm{M}},
$$

where $b=a$ in case of the $\Gamma-\mathrm{X}$ interface and $b=a \sqrt{2}$ in case of the $\Gamma-\mathrm{M}$ interface, provided that the condition $k_{0} b>\pi$ is satisfied simultaneously with Eq. (1).

As follows from the obtained results, the IFCs flatness does not guarantee that $T \approx 1$. In turn, this means that the product of the effective Fabry-Pérot parameters of PC, i.e., $n_{\mathrm{eff}}^{(f)} D_{\text {eff }}$, can depend on $\theta$ in a more complicated fashion than $1 / \cos \theta$. In contrast with negative refraction, ${ }^{14}$ the possibility of using the first $\mathrm{FB}$ wave and $\Gamma-\mathrm{M}$ interfaces in order to obtain an intermediate- $\theta$ passband, which would satisfy Eq. (1), has not been detected. It has been demonstrated that a small- $\theta$ passband and intermediate- $\theta$ passband can simultaneously exist at much smaller $k_{0} a$ than in Figs. 2-4, e.g., at $k_{0} a \approx 1.25$, but conditions (1) are not satisfied. From the comparison of various theoretical performances, it follows that the PC parameters and the range of $k_{0} a$ variation used in Figs. 2-4 are most appropriate.

To summarize, we demonstrated the potential of twodimensional PCs in the obtaining of spatial filters with wide angle ranges of total transmission and relatively steep switching between the ranges of total reflection and total transmission. These ranges are particularly appropriate for the use in bandpass and bandstop filters. They are connected with the FB waves, whose dispersion is characterized by properly located (near-)flat IFCs. However, this does not guarantee that the transmission is total within the wide ranges. Fabry-Pérot type resonances should be carefully adjusted and negative-first-order diffraction taken into account. Best results for the required intermediate- $\theta$ passbands were achieved for a high-contrast PC with the selected lattice parameters and number of the layers.

This work is supported by the European Union under the projects EU-METAMORPHOSE, EU-PHOREMOST, EU-PHOME, and EU-ECONAM, and TUBITAK under the Project Nos. 105E066, 105A005, 106E198, and 106A017. One of the authors (E.O.) also acknowledges partial support from the Turkish Academy of Sciences.

${ }^{1}$ D. Schurig and D. R. Smith, Appl. Phys. Lett. 82, 2215 (2003).

${ }^{2}$ I. Moreno, J. J. Araiza, and M. Avedano-Alejo, Opt. Lett. 30, 914 (2005).

${ }^{3}$ R. Rabady and I. Avrutsky, Opt. Lett. 29, 605 (2004).

${ }^{4}$ A. Sentenac and A.-L. Fehrembach, J. Opt. Soc. Am. A 22, 475 (2005).

${ }^{5}$ L. Dettwiller and P. Chavel, J. Opt. Soc. Am. A 1, 18 (1984).

${ }^{6}$ O. F. Siddiqui and G. Eleftheriades, J. Appl. Phys. 99, 083102 (2006).

${ }^{7}$ A. E. Serebryannikov and T. Magath, J. Opt. Soc. Am. B 25, 286 (2008).

${ }^{8}$ J. H. Wu, L. K. Ang, A. Q. Liu, H. G. Teo, and C. Lu, J. Opt. Soc. Am. B 22, 1770 (2005).

${ }^{9}$ Y. Xiang, X. Dai, S. Wen, and D. Fan, J. Opt. Soc. Am. A 24, A28 (2007)

${ }^{10}$ H. T. Jiang, H. Chen, H. Q. Li, Y. W. Zhang, and S. Y. Zhu, Appl. Phys. Lett. 83, 5386 (2003).

${ }^{11}$ P. A. Belov, C. R. Simovski, and P. Ikonen, Phys. Rev. B 71, 193105 (2005).

${ }^{12}$ E. Cubukcu, K. Aydin, E. Ozbay, S. Foteinopolou, and C. M. Soukoulis, Phys. Rev. Lett. 91, 207401 (2003).

${ }^{13}$ E. Cubukcu, K. Aydin, E. Ozbay, S. Foteinopolou, and C. Soukoulis, Nature (London) 423, 604 (2003).

${ }^{14}$ C. Luo, S. G. Johnson, J. D. Joannopoulos, and J. B. Pendry, Phys. Rev. B 65, 201104 (2002). 